\title{
Thermotropic Behaviour of Liquid Crystalline Poly[n-(4'- cyanophenyl-4"'-phenoxy)alkyl acrylate] Gels in the Dry and Swollen States
}

\author{
Richard Vendamme, ${ }^{12^{*}}$
}

1 Max-Planck-Institut für Polymerforschung, Postfach 3148, D-55021 Mainz, Germany.

$2^{*}$ Université des Sciences et Technologies de Lille, Laboratoire de Chimie Macromoléculaire, Bâtiment C6, CNRS UPRESA No. 8009, F-59655 Villeneuve d'Ascq Cedex, France.

Present address: Frontier Research System (FRS), The Institute of Physical and Chemical Research (RIKEN), Hirosawa 2-1, Wako-shi, Saitama, 351-0198 Japan; fax : (048)-467-9599 ; email : vendamme@riken.jp

(Received: 22 September, 2007; published: 28 November, 2007)

\begin{abstract}
This paper reports the thermotropic behaviour of various side-chain liquid crystalline (LC) networks and gels of poly[n-(4'-cyanophenyl-4"'-phenoxy)alkyl acrylate]. Increasing the aliphatic spacer length tend to increase the melting temperature of the pure LC polymers. On the other hand, increasing the crosslinking density lead to a decrease and broadening of the melting transition temperature. Swelling of a liquid crystalline elastomer in a low molar weight liquid crystal (LMWLC) leads to the formation of a LC gel, with thermotropic properties drastically different from the original LC polymers and solvents. The thermotropic behaviour of such an LC gel is determined by several parameters, such as the concentration of LMWLC inside the gel and the compatibility between the two types of mesogens.
\end{abstract}

\section{Introduction}

Low molecular weight liquid crystals (LMWLCs) are fluids with defined directionality. Cross-linked polymer chains form rubber, a soft solid that is locally liquid-like and capable of huge extension. Liquid crystalline elastomers (LCEs) are a peculiar combination of these two classes of materials, and are defined as loosely crosslinked networks that have rigid-rod, liquid crystalline (LC) molecules incorporated directly into the polymer backbone (i.e., "main-chain" LCEs) or attached to the polymer backbone via a flexible spacer group (i.e., "side-chain" LCEs). It was de Gennes who first theoretically recognized that such organized networks would have unique properties [1]. Subsequently, a huge number of experimental work on LCE has revealed the existence of many remarkable phenomena arising from the strong coupling between the orientational order and mechanical strain [2], e.g., spontaneous shape changes at LC phase transitions [3-5], large opto-mechanical response [6,7], stress-induced macroscopic orientation [8], and unique dynamic mechanical properties [9-11]. The physical properties of linear side-chain liquid crystalline polymers (LCP) are typically determined by the stiffness of the backbone [12-15], the nature of the mesogenic side-groups, and the length of the aliphatic spacer [16-18]. 
In the case of LCEs, additional parameters such as the cross-linking density have also to be considered [19-21].

Recently, liquid crystalline gels (LCG) made of a LCE swollen with a LMWLC have been introduced as a new type of electrically driven soft actuators [22-27]. The advantages of such systems stem from the rapid orientation of the LMWLC in response to an electric field and the possibility to transmit efficiently the orientation to the network at a macroscopic scale, via anisotropic coupling of the mesogenic species. The physical properties of a LCG are determined by the structure of the LCE, but also by the concentration and nature of the LMWLC, as well as by the subtle and complex interplay between these two species. As the physical properties of both LCE and LCG critically depend on their thermotropic behaviour, there is a huge interest to determine how the different structural parameters can affect the phase diagrams of such complex liquid crystalline systems, both in the dry and swollen states.

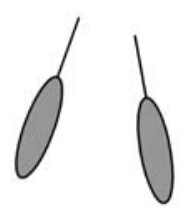

(a)

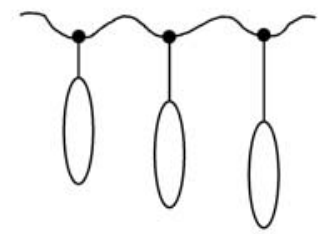

(b)

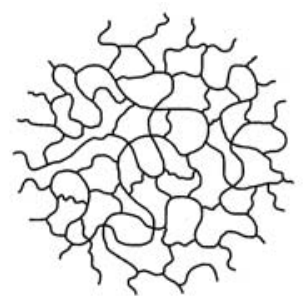

(c)

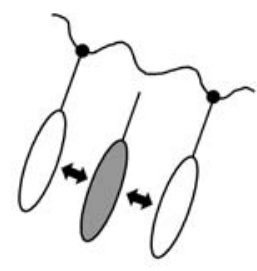

(d)

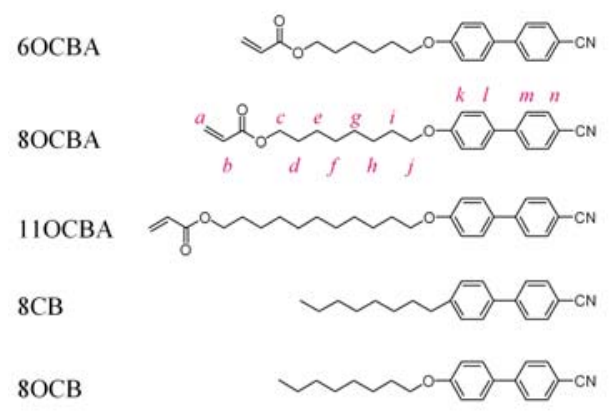

(e)

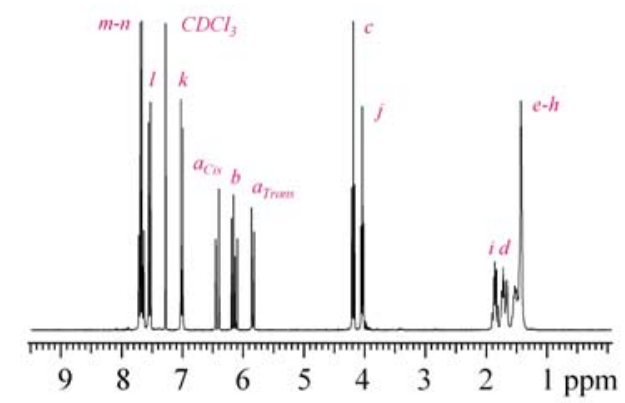

(f)

Fig. 1. Schematic illustrations of the different polymer systems involved in this work: (a) Low molecular weight liquid crystal (LMWLC); (b) Linear side-chain polymer liquid crystal (LCP) with different aliphatic spacer lengths; (c) Cross-linked 3D macromolecular structure characteristic of a liquid crystalline elastomer (LCE); (d) Liquid crystalline gel (LCG) composed of a LCE swollen by a LWMLC. (e) Molecular structures of the employed mesogenic monomers with three different aliphatic spacer lengths and the low molecular weight liquid crystals $8 \mathrm{CB}$ and $80 \mathrm{OB}$. (f) ${ }^{1} \mathrm{H}$ NMR spectra of $80 \mathrm{OBA}$ recorded on a Bruker 300 AMX spectrometer and referenced to the solvent peak (300 $\mathrm{MHz}, \mathrm{CD}_{2} \mathrm{Cl}_{2}$ ).

In the present study, the liquid crystalline properties of various poly[n-(4'cyanophenyl-4"'-phenoxy)alkyl acrylate] are scrutinized. Firstly, the influence of molecular parameters (aliphatic spacer length and cross-linking density) on the thermotropic behaviour of the dry LCEs is considered. Then, the influence of a small amount of LMWLC homogeneously distributed within the LCE is foreseen. In the case of LCG, several parameters, such as the concentration of LMWLC inside the gel 
and the miscibility between the two types of mesogens, are discussed. Schematic illustrations of the different macromolecular systems involved in this work are displayed on Figures 1a)-1d).

\section{Results and Discussion}

\section{Thermotropic Behaviour of Dry Liquid Crystalline Polymers}

\section{-Effect of Spacer Length}

Representative DSC thermograms of the different linear and cross-linked liquid crystalline polymers are presented in Figures $2 a$ ) and $2 b$ ), respectively. All the thermograms of Figure 2a) display a glass transition and a single melting transition, independent of the aliphatic spacer length. In the case of linear LCP, an increase in the spacer length leads to a proportional increase in the melting temperature and simultaneously to a proportional diminution of the glass transition temperature (Figure $2 a)$. The aliphatic spacer affects the viscoelasticity of the polymer backbone, and consequently the glass transition temperature of the system. A short spacer will decrease the mobility of the backbone (increase in glass transition temperature) because of the strong coupling with the rigid mesogenic groups, whether a longer spacer will leave more degrees of freedom to the polymer chain (decrease on the glass transition) and tend to decouple the polymer chain from the liquid crystalline side-chains. The spacer also influences the LC ordering of the mesogenic group. A longer spacer will allow a greater degree of organisation of the mesogens and favour the apparition of layered structures whether, with a short spacer, the mesogenic units are influenced by the steric constraints imposed by the main chain.

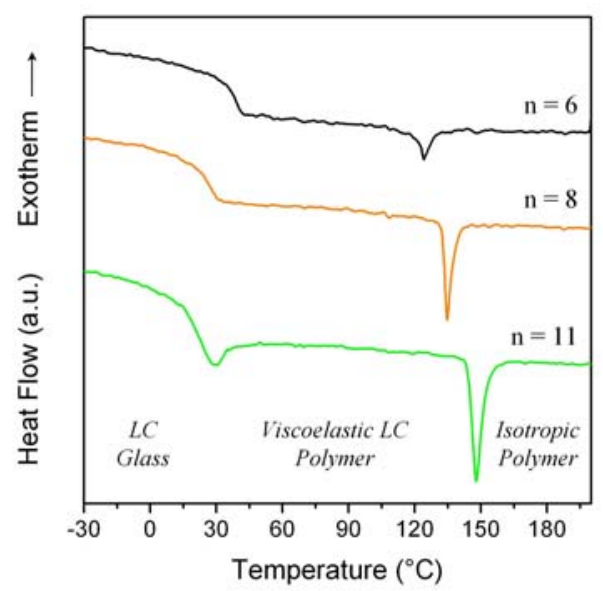

(a)

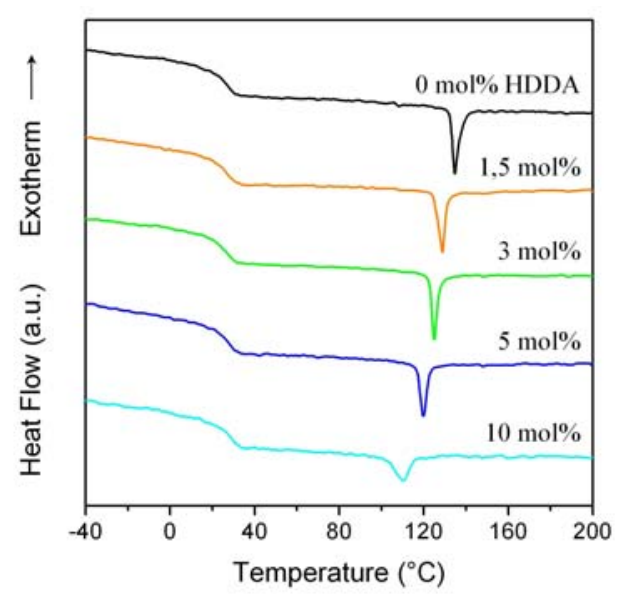

(b)

Fig. 2. (a) Influence of the number $n$ of methylene units in the aliphatic spacer on the normalized differential scanning calorimetry traces $\left(5{ }^{\circ} \mathrm{C} / \mathrm{min}\right)$ of LCP-nOCBA. (b) Effect of cross-linking density on the normalized differential scanning calorimetry traces $\left(5^{\circ} \mathrm{C} / \mathrm{min}\right)$ of LCE-8OCBA specimens.

The melting transition temperature of LCP-8OCBA is $135.6^{\circ} \mathrm{C}$, i.e., $55^{\circ} \mathrm{C}$ higher than the melting transition temperature of the pure LMWLC $80 \mathrm{CB}\left(80.6{ }^{\circ} \mathrm{C}\right)$. Therefore, the polymer backbone suppresses the crystallisation of the $80 \mathrm{CB}$ side chains and significantly stabilise the liquid crystal order over a wide range of temperature. LCP6OCBA, LCP-8OCBA, and LCP-110CBA are isotropic polymers above the melting 
transition temperature, viscoelastic liquid crystalline polymers between the melting transition temperature and the glass transition temperature, and liquid crystalline glasses below the glass transition temperature. Interestingly, the melting transitions of the three linear LCP are relatively sharp (Figure 2a), although their polydispersity is quite large. This observation was surprising since a broad polydispersity is generally believed to cause broad phase transitions [29, 30]. However, in the case of high molecular masses polymers, it has already been shown that the effect of the polydispersity is less pronounced than in the case of low molecular mass liquid crystalline polymers or oligomers [31,32].

\section{-Effect of Cross-Linking Density}

Thermograms of LCE-8OCB specimens are presented on Figure 2b). Evolutions of the glass transition temperature, calorific capacity, melting transition temperature, and enthalpy of the LCE as a function of the cross-linking density are plotted on Figure 3) and reported in Table 1.

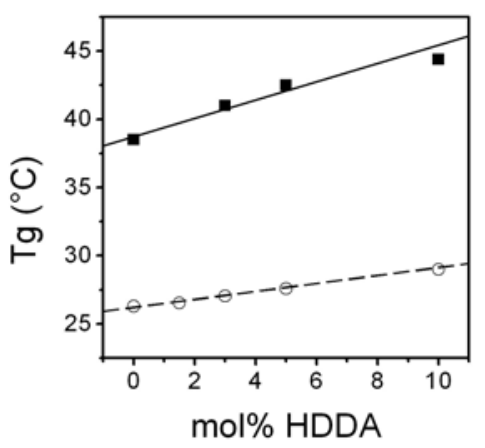

(a)

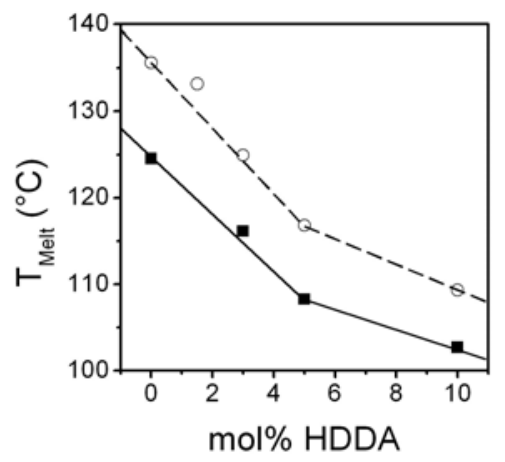

(c)

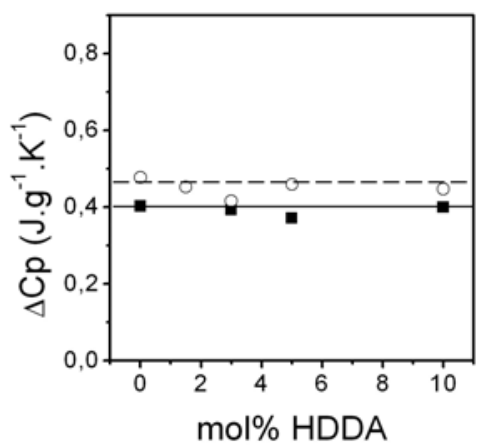

(b)

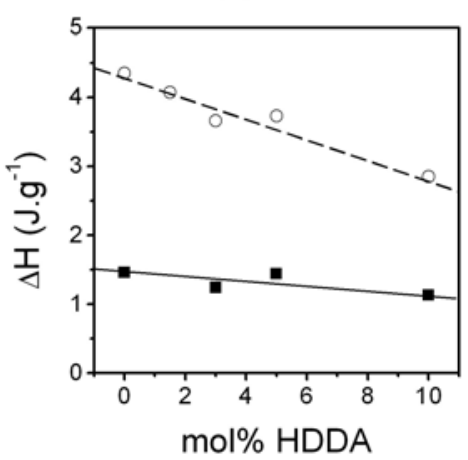

(d)

Fig. 3. Influence of the cross-linking density on the glass transition temperature (a), calorific capacity (b), melting transition temperature (c), and enthalpy (d) for the specimens LCE-6OCBA (symbol ) and LCE-8OCBA (symbolO). Straight lines are guides for the eyes.

The augmentation of the HDDA concentration in the network tend to increase the glass transition temperature and to decrease the melting transition temperature for both LCE-6OCBA and LCE-8OCBA. The augmentation of the glass transition is strictly proportional to the cross-linker density for the two series of networks (Figure 3a). The calorific capacity associated with the glass transition is not affected by the degree of cross-linking (Figure 3b). Both the melting transition temperature and the enthalpies decrease when the HDDA concentration increases. Although the enthalpy 
decreases proportionally as a function of the cross-linker density (Figure $3 d$ ), a different behaviour was observed for the melting temperature (Figure 3c). The melting temperature decreases proportionally with the concentration of HDDA in the concentration range $0 \mathrm{~mol} \%$ to $5 \mathrm{~mol} \%$, but the specimens cross-linked with $10 \mathrm{~mol} \%$ HDDA are clearly beyond the proportional evolution (Figure 3c).

Several factors can be accounted for to explain this observation. First, the crosslinking agent HDDA is a non-mesomorphic molecule and will decrease the concentration of mesogenic groups in the network. Moreover, the HDDA molecules will act as an anchor between two polymer chains and fix the topology of the network in a certain configuration, playing the role of defects for the liquid crystalline ordering. An augmentation of the HDDA concentration will diminish the size and the perfection of the liquid crystalline domains formed by the mesogenic side-chains. The proportionality between the melting transition temperature and the cross-linker concentration in the range $0 \mathrm{~mol} \%$ to $5 \mathrm{~mol} \%$ may indicate that, for such low concentration of HDDA, the cross-linking points are homogeneously distributed within the network. On the other hand, for high concentration such as $10 \mathrm{~mol} \%$, the behaviour observed on Figure $3 \mathrm{c}$ suggests that some phase segregation occurs between HDDA and the mesogenic monomer. This assumption is further supported on Figure $2 \mathrm{~b}$ because the melting transition of the specimen cross-linked with 10 mol\% HDDA is broader than the melting transitions of the loosely cross-linked ones. As the polydispersity influences the breadth of the melting transition, this may imply that the molecular mass of polymer chains between two cross-linking points is not well defined and irregular for the samples cross-linked with $10 \mathrm{~mol} \%$ of HDDA (both LCE-6OCB and LCE-8OCB).

\section{Physical Properties of the Dry Liquid Crystalline Polymers}

Polarized optical microscopy investigations were performed by sandwiching small quantities of linear polymers between two clean glass slides. The samples were then annealed in the isotropic phase $\left(20^{\circ} \mathrm{C}\right.$ above the melting transition temperature $)$ for 30 minutes, and the temperature was slowly cooled with a ramp of $-0.5^{\circ} \mathrm{C} / \mathrm{min}$. Three different textures were observed as a function of the aliphatic spacer length.
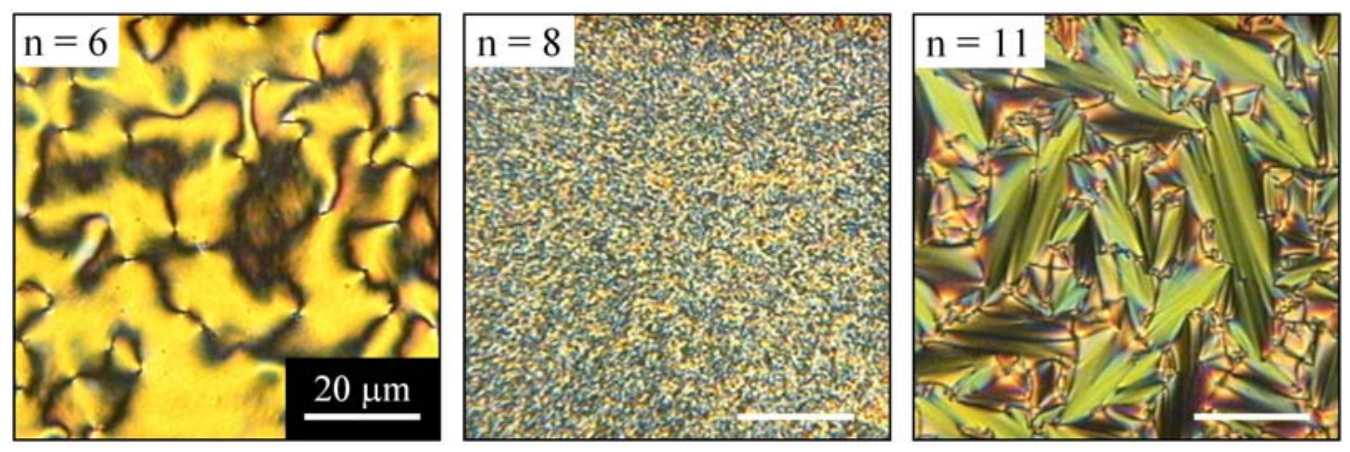

Fig. 4. Polarized optical micrographs (in crossed polarizers) of LCP-6OCBA, LCP8OCBA, and LCP-110CBA.

LCP-6OCBA displays the Schlieren texture characteristics of the nematic phases, whereas the polymer with the longest spacer LCP-110CBA exhibits a fan-shaped focal conic texture typical of low molecular smectic A (Figure 4) [33]. On the other end, the polymer with the intermediate spacer length $(n=8)$ did not present any wellknown texture, and is only birefringent below the melting temperature. Therefore, the 
LC phase of LCP-8OCBA does not correlate with its low molecular weight analogue $80 C B$, which shows both a nematic and smectic textures under the optical microscope. All the cross-linked samples, both LCE-6OCBA and LCE-8OCBA, show are only birefringence and do not display any characteristic textures.

Wide angle X-ray scattering (WAXS) patterns obtained for bulk oriented and nonoriented samples are used to reveal details concerning the ordering on the molecular level. The wide-angle X-ray diffraction 2D patterns of an oriented network of LCE$80 \mathrm{CBA}$ ( $5 \mathrm{~mol} \% \mathrm{HDDA}$ ) recorded at room temperature is shown in Figure $5 \mathrm{a}$. The Xray diffraction intensity distribution of the oriented network as a function of the scattering vector is presented in Figure $5 \mathrm{~b}$. All the polymers and elastomers display the same type of X-ray patterns composed of two rings, independent of the aliphatic spacer length. For non-oriented samples, these two rings are uniform and are characteristic of a polydomain LC structure.

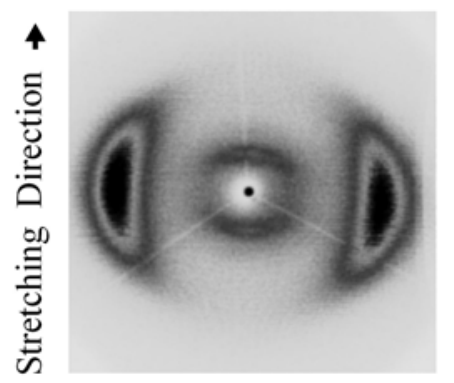

(a)

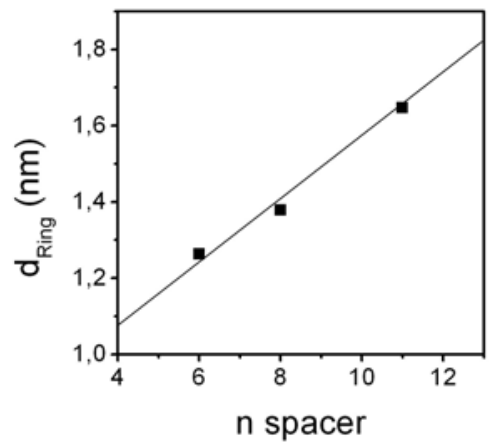

(c)

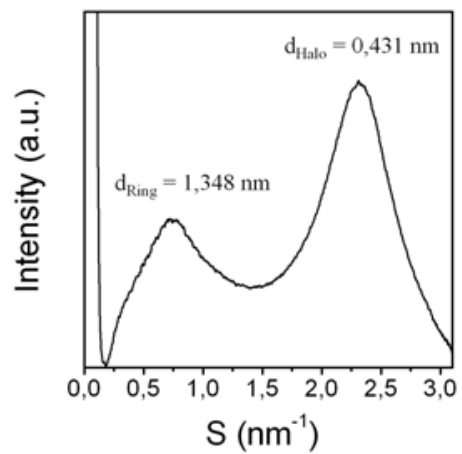

(b)

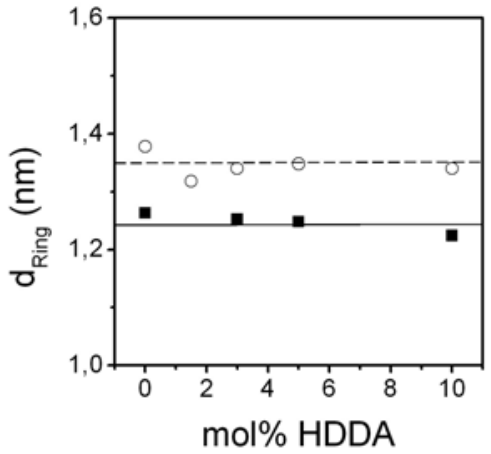

(d)

Fig. 5. X-ray diffraction 2D patterns of a LCE-8OCBA (5 mol\% HDDA) network in the oriented state (a). X-ray diffraction intensity distribution versus the scattering vector $S$ recorded for the oriented LCE-8OCBA (5 mol\% HDDA) network at room temperature (b). Evolution of the distance corresponding to the inner ring as a function of the number $\mathrm{n}$ of carbon in the aliphatic spacer for the linear LCPs (c) and as a function of the cross-linking density (d) for the specimens LCE-6OCBA (symbol a) and LCE8OCBA (symbol O).

The outer ring (halo corresponding to high scattering vector) corresponds to the lateral spacing of the mesogens and do not depend on the aliphatic spacer length or cross-linking density, as shown in Table 1 . The inner ring is very diffuse for LCP6OCBA and LCE-6OCBA, diffuse for LCP-8OCBA and LCE-8OCBA, but is more pronounced for LCP-110CBA. The inner ring of LCP-110CBA is attributed to the distance between smectic layers $(\mathrm{d}=1.45 \mathrm{~nm})$. The origin of the diffuse inner ring for 
LCP-6OCBA and LCP-80CBA is, however, more difficult to determine. Selforganisation of the mesogenic side-groups lead to the formation of liquid crystalline aggregates that segregate from the aliphatic segments (backbone + spacer). In the case of oriented specimens, the mesogens are oriented parallel to the stretching direction. The distances corresponding to the inner ring of the X-ray patterns are attributed to average distances of periodicity between liquid crystalline aggregates and aliphatic segments. Those distances are proportional to the spacer length (Figure 5c) but do not depend on the cross-linking density (Figure 5d).

Tab. 1. Physical properties of the dry LC materials prepared in this study. The indicated parameters are: the cross-linking density $\left(C_{x}\right.$ in HDDA mol\%), the glass transition temperature $\left(\mathrm{T}_{\mathrm{g}}\right.$ in $\left.{ }^{\circ} \mathrm{C}\right)$, the calorific capacity $\left(\Delta \mathrm{Cp}\right.$ in $\left.\mathrm{J} \cdot \mathrm{g}^{-1} \cdot \mathrm{K}^{-1}\right)$, the melting transition temperature $\left(\mathrm{T}_{\text {Melt }}\right.$ in $\left.{ }^{\circ} \mathrm{C}\right)$, the enthalpy $\left(\Delta|\mathrm{H}|\right.$ in $\left.\mathrm{J}^{-g^{-1}}\right)$, the modulus recorded at $137{ }^{\circ} \mathrm{C}\left(\mathrm{E}^{\prime}\right.$ in $\left.\mathrm{Pa}\right)$, and the distances (in $\mathrm{nm}$ ) corresponding to the outer ring $\left(\mathrm{d}_{\text {Halo }}\right)$ and inner ring $\left(\mathrm{d}_{\text {Ring }}\right)$ observed on the X-ray diffraction $2 \mathrm{D}$ patterns.

\begin{tabular}{|c|c|c|c|c|c|c|c|c|}
\hline \multicolumn{2}{|c|}{ Sample } & \multicolumn{4}{|c|}{ DSC } & \multirow{2}{*}{$\frac{\text { DMA }}{E^{\prime}}$} & \multicolumn{2}{|c|}{ WAXS } \\
\hline Name & $C_{x}$ & $\mathrm{~T}_{\mathrm{g}}$ & $\Delta \mathrm{Cp}$ & $\mathrm{T}_{\text {Melt }}$ & $\Delta|H|$ & & $\mathrm{d}_{\text {Halo }}$ & $\mathrm{d}_{\text {Ring }}$ \\
\hline LCP-60CBA & 0 & 38.5 & 0.40 & 124.5 & 1.46 & - & 0.425 & 1.263 \\
\hline LCE-6OCBA & 3 & 41.0 & 0.39 & 116.1 & 1.24 & 5500 & 0.434 & 1.252 \\
\hline LCE-6OCBA & 5 & 42.5 & 0.37 & 108.2 & 1.44 & 9500 & 0.432 & 1.248 \\
\hline LCE-6OCBA & 10 & 44.4 & 0.39 & 102.7 & 1.13 & 21200 & 0.434 & 1.224 \\
\hline LCP-80CBA & 0 & 26.3 & 0.47 & 135.6 & 4.35 & - & 0.426 & 1.378 \\
\hline LCE-8OCBA & 1.5 & 26.5 & 0.45 & 133.2 & 4.07 & - & 0.429 & 1.318 \\
\hline LCE-8OCBA & 3 & 27.0 & 0.41 & 124.9 & 3.66 & 6200 & 0.430 & 1.340 \\
\hline LCE-8OCBA & 5 & 27.6 & 0.45 & 116.8 & 3.73 & 9800 & 0.431 & 1.348 \\
\hline LCE-8OCBA & 10 & 29.0 & 0.44 & 109.3 & 2.85 & 19500 & 0.434 & 1.340 \\
\hline LCP-110CBA & 0 & 22.0 & 0.51 & 147.9 & 7.32 & - & 0.427 & 1.647 \\
\hline
\end{tabular}

Evolutions of the storage modulus E' (energy storage) and the loss modulus E" (energy loss) as a function of temperature were plotted in Figure 6 for LCP-8OCBA and LCE-8OCBA ( $5 \mathrm{~mol} \% \mathrm{HDDA}$ ). The glass transition and the melting transition can be clearly identified on those rheograms for both LCP-8OCB and LCE-8OCB. The glass transition temperature corresponds to a specific transition where the polymer changes from a glassy state with high modulus to a viscoelastic condition and is clearly observed for both the linear and cross-linked polymer around $25{ }^{\circ} \mathrm{C}$. The evolution of the rheograms of the linear and cross-linked samples around the glass transition temperature did not present fundamental differences.

However, around the melting transition temperature, the cross-linked architecture induces tremendous distortion on the rheogram of the LCE, as compared to that of the LCP. The melting transition are characterized by abrupt steps for both E' and E" (Figure 6) [9]. Above $T_{\text {Melt, }}$ the rheogram of LCP-8OCBA shows a viscous flow (E" $>E$ ') characteristic of a fluid behaviour, whereas the rheogram of LCE-8OCBA (5 mol\% HDDA) presents a rubbery plateau (E' quasi constant and low E") similar to the plateau observed for conventional vulcanized rubbers. 


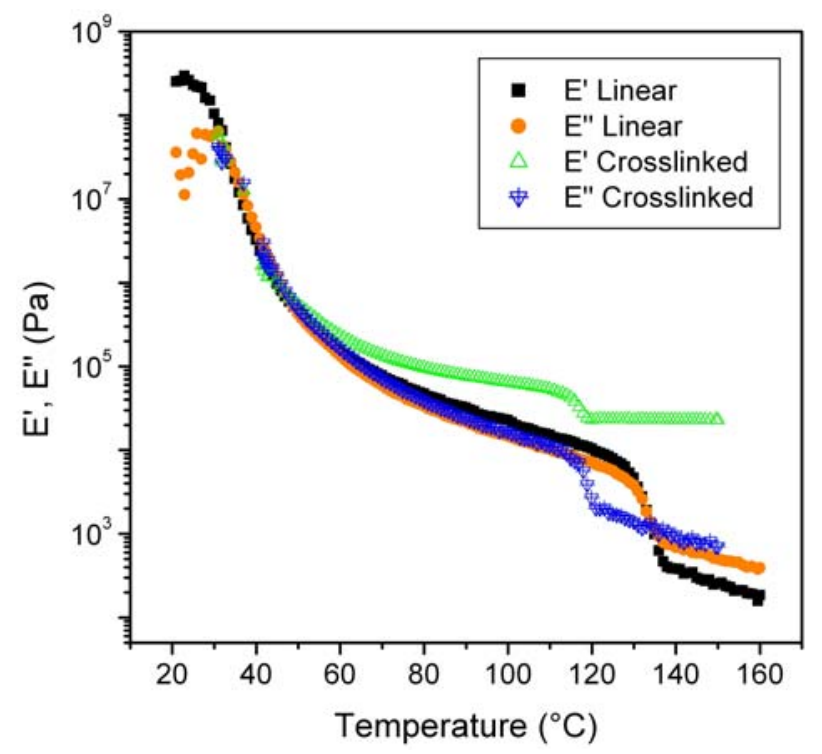

Fig. 6. Evolution of the storage modulus E', and loss modulus E' as a function of temperature for the linear polymer LCP-8OCBA and the network LCE-8OCBA (5 mol\% HDDA). The measurements were performed in the linear viscoelastic regime, i.e., the storage and loss moduli were independent of the imposed strain amplitude.

The melting temperatures of LCP-8OCBA and LCE-8OCBA (5 mol\% HDDA) can be determined from the midpoints of the transition ranges on the rheograms and were determined as $133{ }^{\circ} \mathrm{C}$ and $116{ }^{\circ} \mathrm{C}$, respectively, in very good agreement with the DSC values of the same materials $\left(136{ }^{\circ} \mathrm{C}\right.$ and $117{ }^{\circ} \mathrm{C}$, respectively). Similar rheograms were obtained with LCE-8OCBA of different cross-linking densities and also for LCE-6OCBA specimens. However, it should be noted that the melting transition could not be detected on the rheograms of LCE-6OCBA (no step in the rheograms for both E' and E'). Young moduli of the LCEs were recorded at $137{ }^{\circ} \mathrm{C}$ (in the rubbery plateau) and are reported in Table 1. The modulus of the network increases with the cross-linking density in accordance with the theory of rubber elasticity [34, 35].

\section{Thermotropic Behaviour of Liquid Crystalline Gels}

\section{-Temperature / Concentration Phase Diagram in the Full Concentration Range}

The swelling behaviour of LC network in LC solvents has been the subject of many theoretical studies since several decades $[36,40]$ but surprisingly has attracted experimental investigations only recently. Urayama first reported the equilibrium swelling behaviour of LC networks in nematic [41, 42] and isotropic [43] solvents and show that the principal features of the swelling can be understood with a mean field theory. Yusuf et al. studied the dynamic swelling behaviour of dry poly-domain or mono-domain LC elastomer in nematic solvent [44]. We reported very recently a comparative study of the swelling behaviour of isotropic networks of poly(n-butyl acrylate) and nematic LCE-6OCBA networks swollen by nematic LMWLCs (5CB, $7 \mathrm{CB}$, and $\mathrm{E} 7$ ), and showed experimentally that the nematic coupling between the free mesogens and the mesogenic side-groups induced tremendous distortion on the phase diagrams of binematic mixture with a cross-linked architecture [45].

A piece of LCE-8OCBA (5 mol\% HDDA) was allowed to swell freely in the smectogenic LMWLC 8CB. The gel always swells homogeneously in all directions, 
even in the liquid crystalline state, because of the polydomain structure of the gel, which annihilates the local LC ordering on a macroscopic scale. Unidirectional swelling degree $\lambda$ of the gel was determined by optical microscopy, as described earlier [45-46], and is displayed in Figure 7a. The swelling of LCE-8OCBA in 8CB is strongly correlated to the phase of $8 \mathrm{CB}$ inside and outside the gel. Below $\mathrm{T}_{\mathrm{Nl}}{ }^{8 \mathrm{CB}}$ (temperature of nematic to isotropic transition), the swelling degree increases linearly with temperature (any appreciable volume change is not observed around $\mathrm{T}_{\mathrm{SN}}{ }^{8 \mathrm{CB}}$ ).

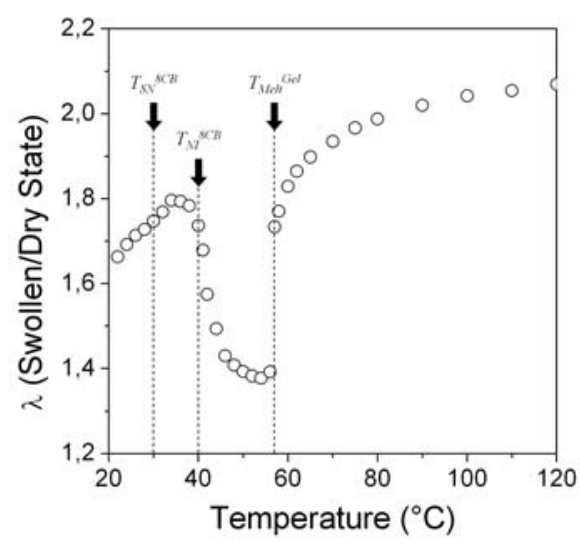

(a)

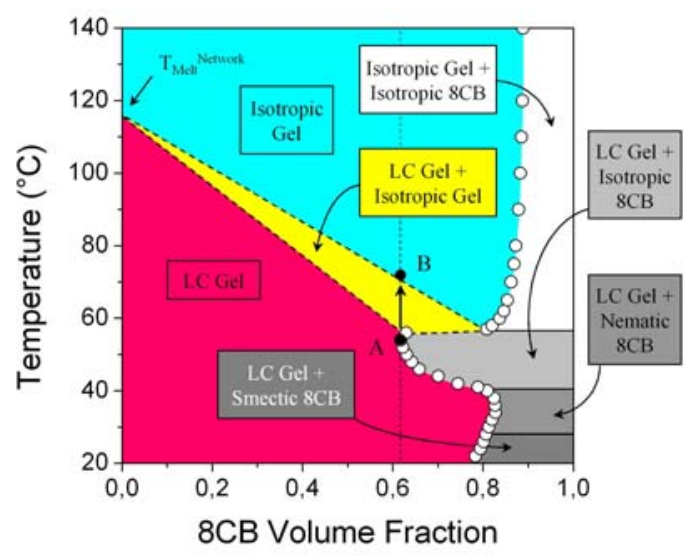

(b)

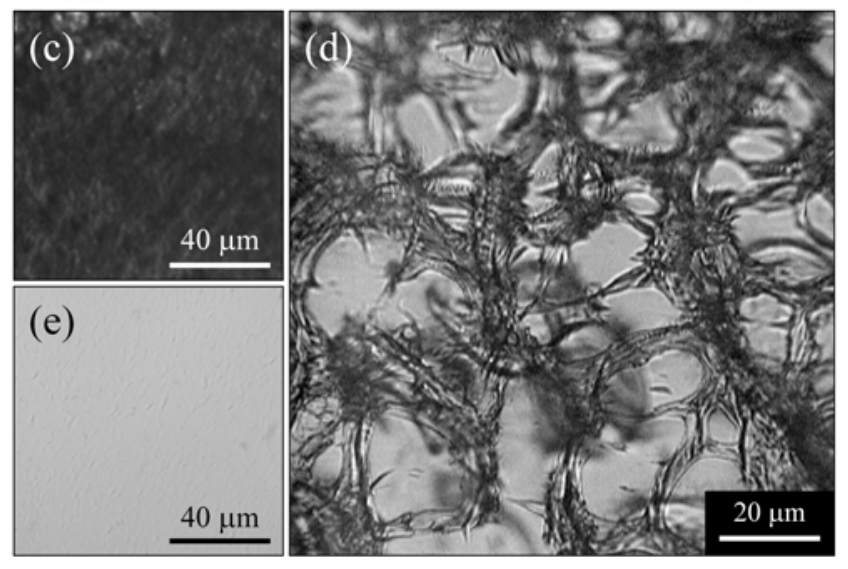

Fig. 7. (a) Unidirectional swelling degree as a function of the temperature for a LCE8OCBA (5 mol\% HDDA) network swollen in 8CB. (b) Concentration / temperature phase diagram of $8 \mathrm{CB}$ inside the LCE-8OCBA network. The signification of the points $A$ and $B$ is given in the text. C-e) Thermotropic behaviour of LCE-8OCBA (5mol\% HDDA)/8CB gel with $\Phi_{8 \mathrm{CB}}=0.61$; (c) Optical image (parallel polarizers) of the homogeneous liquid crystalline gel phase at $53^{\circ} \mathrm{C}$. The gel is birefringent and becomes bright under crossed polarizers. (d) Optical image (parallel polarizers) showing the coexistence of a nematic gel and an isotropic gel at $66^{\circ} \mathrm{C}$. The fibril-like dark domains are birefringent and become bright under crossed polarizers, whether the clearer domains are isotropic. (e) Optical image (parallel polarizers) of the fully isotropic gel at $73^{\circ} \mathrm{C}$.

At $\mathrm{T}_{\mathrm{Nl}}{ }^{8 \mathrm{CB}}, 8 \mathrm{CB}$ becomes isotropic whether the gel remains liquid crystalline. Further increase in the temperature depresses the strength of anisotropic interactions inside the gel phase and results in the gel collapsing. As noted by Urayama [41, 42], the transition of the gel from the liquid crystalline to isotropic state at $\mathrm{T}_{\text {Melt }}{ }^{\text {Gel }}$ induces a large discontinuous volume phase transition. Above $\mathrm{T}_{\text {Melt }}$ Gel the isotropic network is not saturated and continues to absorb some isotropic $8 \mathrm{CB}$. 
The swelling degree in volume of the gel is $Q=\lambda^{3}=1 / \Phi_{\text {Network}}$, where $\Phi_{\text {Network }}$ is the volume fraction of polymer in the gel phase. The volume fraction of $8 \mathrm{CB}$ in the gel is defined as $\Phi_{8 \mathrm{CB}}=1-\Phi_{\text {Network }}=1-\left(1 / \lambda^{3}\right)$. The coexistence curves (binodals) of the phase diagrams can be constructed by plotting $\Phi_{8 \mathrm{CB}}=f(T)$. The concentration/temperature phase diagram of the LCE-8OCBA/8CB system is presented in Figure $7 \mathrm{~b}$ and can be divided into seven domains. The thermotropic behaviours of pure LCE-8OCBA and pure 8CB correspond to the vertical lines $\Phi_{8 \mathrm{CB}}=$ 0 and $\Phi_{8 C B}=1$, respectively. Between these two extreme cases, a wide variety of phases are observed as a function of the temperature and the concentration of $8 \mathrm{CB}$. The four domains on the right of the coexistence curve correspond to a biphasic system composed of a swollen gel (either isotropic or LC) and an excess of 8CB (either smectic, nematic or isotropic). The gel phase is always homogeneous because the elasticity of the network suppresses excessive swelling, which results in phase separation between the swollen network and the pure solvent [47-49]. On the left side of the binodal, three domains are distinguished: a homogeneous liquid crystalline gel, a homogeneous isotropic gel, and a zone of coexistence between an isotropic gel and a liquid crystalline gel. A peculiar behaviour of the phase diagram of a LCE swollen by a LMWLC is the wide miscibility gap between the melting transition temperature of the pure solvent $\left(\mathrm{T}_{\mathrm{NI}}{ }^{8 \mathrm{CB}}\right)$ and the melting transition temperature of the gel $\left(T_{\text {Melt }}{ }^{\text {Gel }}\right)$. The exact nature of the liquid crystalline phase of the LCE-8OCBA/8CB gel as a function of temperature is not determined at that time and will be the subject of further studies. A gel showing birefringence under the polarized microscope was simply termed liquid crystalline.

\section{Thermotropic Behaviour of a Liquid Crystalline Gel at Fixed Concentration}

We were interested in the present study to determine the thermotropic behaviour of a LCE swollen with a fixed concentration of LMWLC. To do so, the following experiment was performed. A small piece of LCE-8OCBA (5 mol\% HDDA) was equilibrated in $8 \mathrm{CB}$ at $53{ }^{\circ} \mathrm{C}\left(\Phi_{8 \mathrm{CB}} \approx 0.61\right)$, and subsequently removed from the excess of solvent. The excess of $8 \mathrm{CB}$ on the gel surface was removed by adsorption with a paper. As the network was swollen at equilibrium in the miscibility gap of the phase diagram (point $A$ on Figure $7 b$ ), heating or cooling the gel will always lead to an unsaturated gel. This gel was placed under a polarised optical microscope and heated from $30{ }^{\circ} \mathrm{C}$ to $100^{\circ} \mathrm{C}$ (heating ramp $0,5{ }^{\circ} \mathrm{C} / \mathrm{min}$ ). Three stable phases were observed. From $30{ }^{\circ} \mathrm{C}$ to $55{ }^{\circ} \mathrm{C}$, a homogeneous, birefringent liquid crystalline gel phase is observed (Figure 7c). Between $56{ }^{\circ} \mathrm{C}$ and $72{ }^{\circ} \mathrm{C}$, a progressive phase transition from the liquid crystalline state to the isotropic state is observed. In that temperature range, the gel is biphasic and we observed fibril-like birefringent domains surrounded by an isotropic medium (Figure 7d). The birefringent domains progressively disappeared as the temperature is raised. Finally, the gel becomes fully isotropic around $72{ }^{\circ} \mathrm{C}$ (point $\mathrm{B}$ in Figure $7 \mathrm{~d}$ ) as shown in Figure 7e. The melting transition of the LCG is then considerably broadened as compared to the dry LCE. The melting transition of the LCE-8OCBA/8CB gel spans over $16{ }^{\circ} \mathrm{C}$, whether the melting transition of the pure LCE-8OCBA (5 mol\% HDDA) observed in the same conditions (polarized optical microscopy, heating ramp $0.5^{\circ} \mathrm{C} / \mathrm{min}$ ) takes place within approximately $1^{\circ} \mathrm{C}$.

It is important to note that the biphasic gel structure observed on Figure $7 d$ is thermodynamically stable and do not stem from a gel syneresis (no phase separation could occur inside the gel because the quantity of LMWLC is insufficient to saturate the network at this temperature) but from the supramolecular organisation of the gel. 
Some polymer rich domains inside the LCG may have a higher melting temperature because of the higher melting transition temperature of the side-group polymer network. On the other hand, 8CB-rich domains should have a lower melting temperature. The presence of such polymer-rich and solvent-rich domains may arise from the relative miscibility of the LMWLC with the mesogenic side-groups of the polymer, and also from the structure of the dry LCE itself. In fact, the development of radically cross-linked polymer networks usually proceeds via the formation of microgels and the subsequent percolation of those microgels to give a macroscopic 3D structure. Such a mechanism creates spatial heterogeneities in the cross-linking density within the whole network [50]. Such heterogeneities in the dry LCE should certainly affect the structure of the (isotropic gel + LC gel) biphasic phase. Analysis of Figure $7 \mathrm{~b}$ reveals that the broadening of the melting transition of the LCE-8OCBA ( 5 mol\% HDDA) network swollen with a fixed concentration of $8 \mathrm{CB}$ will increase proportionally with the concentration of $8 \mathrm{CB}$ in the gel, but the system will encounter phase separation during the heating/cooling processes if the concentration of $8 \mathrm{CB}$ within the gel is greater than 0.61 .

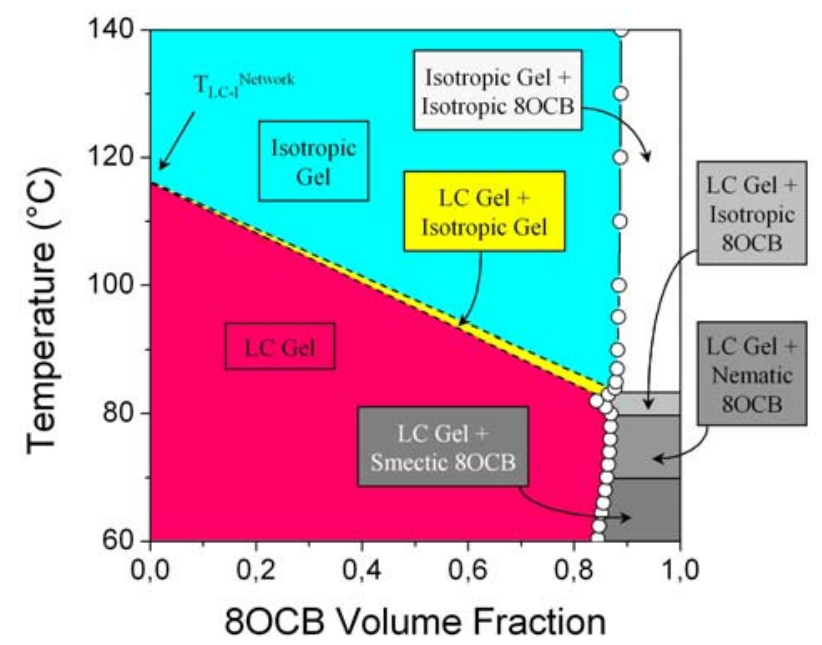

Fig. 8. Concentration / temperature phase diagram of the LCE-8OCBA $(5 \mathrm{~mol} \%$ HDDA)/8OCB gel in the full concentration range. The "LC Gel + Isotropic Gel" chimney in this phase diagram (zone in yellow) is narrower than for the LCE8OCBA/8CB system (see Figure 7b).

It has already been reported that the melting transition of a mixture of a linear sidegroup nematic LCP and its LMWLC analogue is broader than the melting transition of the pure polymer [51,52]. During the melting transition of such mixtures, the system is typically composed of nematic polymer-rich droplets dispersed in an isotropic LMWLC-rich medium, and is consequently composed of two separate phases (one nematic and one isotropic) [51]. On the other hand, the melting transition of a LCE swollen with a LMWLC occurs without macroscopic phase separation. The structure observed in Figure $7 \mathrm{~d}$ is a peculiarity of topologically cross-linked systems, and originates from the competition between the elasticity of the macromolecular structure and the demixing of polymer-rich and LMWLC-rich domains.

The concentration/temperature phase diagram of LCE-8OCBA (5 mol\% HDDA) in $80 C B$ display the same general features (Figure 8), although the shape of the coexistence curves and the melting transition of the gels were quite different from the LCE-8OCBA/8CB system. Especially, we note that the miscibility gap between isotropic $8 \mathrm{OCB}$ and the liquid crystalline gel proceeds within a very narrow 
concentration range, contrasting with the large concentration range observed in Figure $7 \mathrm{~b}$ with the $8 \mathrm{CB}$ system. It is interesting to note that the network's concentration inside the gel is very similar for the two systems in the fully isotropic zone (both gel isotropic and excess of solvent isotropic) and in the fully liquid crystalline zone (LC gel with an excess of LC solvent). The coexistence domain between the isotropic gel and the liquid crystalline gel is narrower for the $80 \mathrm{CB}$ system than for the 8CB system. Therefore, for LCE-8OCBA networks swollen with a given concentration of LMWLC (8OCB or $8 \mathrm{CB}$ ), the melting transition of the LCE8OCBA/8OCB gel will be sharper than the melting transition of the LCE-8OCBA/8CB gel. These differences can be accounted for by the different thermotropic properties of $80 \mathrm{CB}$ as compared to $8 \mathrm{CB}\left(\mathrm{T}_{\mathrm{Nl}}{ }^{80 \mathrm{CB}}>\mathrm{T}_{\mathrm{Nl}}{ }^{8 \mathrm{CB}}\right.$; and consequently $\left\{\mathrm{T}_{\text {Melt }}\right.$ etwork $\left.\mathrm{T}_{\mathrm{NI}}{ }^{8 \mathrm{OCB}}\right\}<\left\{\mathrm{T}_{\text {Melt }}\right.$ Network $\left.-\mathrm{T}_{\mathrm{NI}}{ }^{8 \mathrm{CB}}\right\}$ ), but also by the different liquid crystalline arrangements inside the gel. The strong anisotropic interactions between the 80CB molecules and the 8OCB side-group (same chemical structure) would enhance the anisotropic coupling between the network and the solvent. It is also known that matching the structure of the side-groups and LC solvent tend to increase the solubility [53]. On the other hand, the anisotropic coupling of the 8OCB side-chains with $8 \mathrm{CB}$ molecules will be less effective because of their different chemical nature.

\section{Conclusions}

The thermotropic behaviour of poly[n-(4'-cyanophenyl-4"-phenoxy)alkyl acrylate] in the dry state is primarily controlled by the length of the aliphatic spacer and the crosslinking density, reflecting the coupling between the backbone and the mesogenic side-groups. Swelling of the cross-linked LCE with a LMWLC considerably affects the phase behaviour of the system. In particular, we observed the broadening of the melting transition and the stable coexistence between an isotropic gel and a liquid crystalline gel. The broadening of the melting transition of the LCG as compared to dry LCEs depends on several parameters such as the concentration of LMWLC inside the gel and the compatibility between the two mesogens, reflecting the strong anisotropic coupling between the side-groups of the network and the LMWLC. In summary, both molecular and supramolecular parameters affect the thermotropic properties of liquid crystalline, cross-linked polymer systems.

Several research directions can be explored in future studies. Rheological investigations of the liquid crystalline gels with various LMWLC concentrations may help to understand how the self-assembly of a small anisotropic molecules within the LC network affect the physical properties of the system. The influence of the LCE's cross-linking density on the physical and thermotropic behaviour of the LCG should reveal interesting phenomena [54]. Another interesting research direction is to study the influence of the network structure on the thermotropic behaviour of the LCEs and the LCGs. As mentioned earlier in the text, photo-polymerized networks may possess some heterogeneity and consequently the molecular mass between two consecutive cross-links will be polydisperse. On the other hand, LCE elaborated via ATRP should have narrower distribution of $M_{c}$ and well-defined structure. Moreover, Kornfield et al. demonstrated recently the extremely well defined structure of LCG designed from the self-assembly of an ABA triblock copolymer with a side-group liquid crystalline midblock and liquid-crystal-phobic endblocks in a nematic LMWLC [55]. Such type of physical LCG should display interesting thermotropic behaviour; quite different from the conventional chemically cross-linked LCG. 


\section{Experimental Section}

\section{Materials}

The photo-initiator 2-hydroxy-2-methyl-1-phenyl-1-propanone (Darocur 1173) and the cross-linking agent 1,6-hexanediol diacrylate (HDDA) were obtained from Ciba and Aldrich, respectively. The smectic LMWLCs 4-n-octyl-4'-cyanobiphenyl (8CB) and 4n-octyloxy-4'-cyanobiphenyl (8OCB) was purchased from Merck Eurolab GmbH (Darmstadt, Germany). $8 \mathrm{CB}$ is characterized by a crystalline to smectic transition temperature $\mathrm{T}_{\mathrm{CrS}}=22.8^{\circ} \mathrm{C}$, a smectic to nematic transition temperature $\mathrm{T}_{\mathrm{SN}}=33.6{ }^{\circ} \mathrm{C}$ and a nematic to isotropic transition temperature $\mathrm{T}_{\mathrm{NI}}=40.9{ }^{\circ} \mathrm{C}$. For $80 \mathrm{OB}$, these characteristic temperatures are $\mathrm{T}_{\mathrm{CrS}}=55.4{ }^{\circ} \mathrm{C}, \mathrm{T}_{\mathrm{SN}}=68.4{ }^{\circ} \mathrm{C}$ and $\mathrm{T}_{\mathrm{NI}}=80.6{ }^{\circ} \mathrm{C}$, respectively.

\section{Synthesis of Liquid Crystalline Materials}

n-(4'-Cyanophenyl-4"-phenoxy)alkyl acrylate monomers bearing the same oxy cyano biphenyl mesogenic group but different aliphatic spacers were prepared following the synthetic procedure reported in the literature [28]. The structure of the monomers 6OCBA, 8OCBA and 110CBA are illustrated on Figure 1e) and the ${ }^{1} \mathrm{H}$ NMR spectra of $80 \mathrm{OCBA}$ is displayed on Figure 1f).

Several LCE were synthesised from the monomers 6OCBA and 80CBA as follows: $500 \mathrm{mg}$ of the mesogenic acrylic monomer were dissolved in $2 \mathrm{ml}$ of a dimethylsulfoxide-toluene mixture (40-60 vol.\%) [24]. Then, the difunctional molecule HDDA and a small amount of initiator were added to the mixture. This isotropic formulation was stirred mechanically for one hour, introduced into a Teflon mold and exposed to the UV lamp (Philips TL08, wavelength of $350 \mathrm{~nm}$, intensity of 1.5 $\mathrm{mW} / \mathrm{cm}^{2}$ ) under nitrogen atmosphere for 30 minutes. The isotropic gel was transferred into a bath of dichloromethane and the solvent was renewed four times in order to eliminate unattached species. Then, the solvent quality was gradually decreased via successive exchanges in dichloromethane/methanol mixtures. At the end of the process, the collapsed LCE were finally obtained as white discs floating in pure methanol. Subsequently, the networks were dried at $40{ }^{\circ} \mathrm{C}$ for 2 days and then at room temperature under vacuum for one day. The amount of cross-linker in the total reactants introduced during synthesis was varied from $1.5 \mathrm{~mol} \%$ to $10 \mathrm{~mol} \%$. We call this parameter the cross-linking density of the network, although the true cross-linking density is presumably smaller than the cross-linker concentration during synthesis because the cross-linking efficiency will be reduced by the presence of the mesogenic groups. The networks poly[n-(4'-cyanophenyl-4"'-phenoxy)hexyl acrylateco-1,6-hexanediol diacrylate], and poly[8-(4'-cyanophenyl-4"-phenoxy)octyl acrylateco-1,6-hexanediol diacrylate] is abbreviated as LCE-6OCBA, and LCE-8OCBA, respectively.

In addition to the LCEs, linear LC polymers were synthesized by using the same experimental set-up, but without adding HDDA in the initial reactive formulation. The linear polymers were reprecipitated two times from THF $(5 \mathrm{~mL})$ into a warm $\left(50{ }^{\circ} \mathrm{C}\right)$ solution of ethanol $(50 \mathrm{~mL})$, which was cooled to room temperature prior to collecting the precipitates. The LCPs were obtained as white, hard solids and characterized by gel permeation chromatography using THF as eluent. The number average molar masses $\left(M_{n}\right)$ of the polymers poly[n-(4'-cyanophenyl-4"'-phenoxy)hexyl acrylate], poly[8-(4'-cyanophenyl-4"'-phenoxy)octyl acrylate], and poly[11-(4'-cyanophenyl-4"'phenoxy)undecyl acrylate] are 45000, 41000, and 39100, respectively. Those three linear polymers are, however, quite polydisperse as their polydispesity indexes are 
1.98, 1.6, and 1.83, respectively (Gel permeation chromatography measurements were carried out using a Polymer Lab chromatographer with two Ultra Styragel linear columns with pore sizes of $10^{4}$ and $500 \AA$, a UV detector $(254 \mathrm{~nm})$, polystyrene standards and THF as solvent, at $25^{\circ} \mathrm{C}$ with a flow rate of $\left.1 \mathrm{ml} \cdot \mathrm{min}^{-1}\right)$.

\section{Instruments and Methods}

Differential scanning calorimetry (DSC) experiments were conducted with cooling and heating rates of $5 \mathrm{~K} \cdot \mathrm{min}^{-1}$ using a Mettler 30 calorimeter with a cell purged with 50 $\mathrm{ml} / \mathrm{min}$ of nitrogen. Calibration for the temperature and enthalpy changes was performed using Indium as a standard. The second heating run is considered as reflecting the properties of the compact bulk material. Glass transition temperatures were determined from the midpoint of the transition range. Polarized optical microscopy (POM) observations were performed using a Zeiss microscope (D-7082) equipped with a temperature controlled stage (Linkam TMS91/THM600) and a color digital camera (Hitachi KPD50). Wide-angle X-ray diffraction (WAXS) patterns were recorded using the X-ray beam with a pinhole collimation and a $2 \mathrm{D}$ detector (Siemens) with $1024 \times 1024$ pixels. A double graphite monochromator for the CuKa radiation $(\lambda=0.154 \mathrm{~nm})$ was used. The beam diameter was about $0.5 \mathrm{~mm}$ and the sample to detector distance was $80 \mathrm{~mm}$. The recorded scattered intensity distributions were integrated over the azimuthal angle and are presented as functions of the scattering vector $(s=2 \sin \theta / \lambda$, where $2 \theta$ is the scattering angle). Dynamic mechanical analysis was performed using a RMS 800 rhemoeter (Rheometrics Inc). Thermo-mechanical measurements were performed in the linear viscoelastic regime (plate-plate configuration, disk like samples with a diameter of $6 \mathrm{~mm}$ and a thickness of $1.3 \mathrm{~mm}$ ), at a constant frequency of $1 \mathrm{~Hz}$ using the temperature as a variable parameter (heating ramp $2{ }^{\circ} \mathrm{C} / \mathrm{min}$ ).

\section{Acknowledgements}

This work was accomplished during the author's stay at Max-Planck-Institut für Polymerforshung (MPIP) in Mainz (Germany) and made possible by grants from Marie Curie Training Site, Max Planck Research School for Polymer Material Science, and the MPIP. The author is grateful to Dr. Ulrich Maschke for assistance with the fellowships and to Prof. Tadeusz Pakula for enriching scientific discussions. It is a pleasure to thank Dr. Benjamin Carbonnier for having performed most of the WAXS experiments.

\section{References}

[1] de Gennes, P. G. Phys. Lett. 1969, A28, 725.

[2] Gleim, W.; Finkelmann, F. in Side-Chain Liquid Crystal Polymers; McArdle, C. B., Ed., Blackie and Son Ltd.: 1989; p 287.

[3] Brehmer, M.; Zentel, R. Mol. Cryst. Liq. Cryst. 1994, 243, 353.

[4] Mitchell, G. R.; Davis, F. J.; Guo, W. Phys. Rev. Lett. 1993, 71, 2047.

[5] Küpfer, J.; Finkelmann, H. Makromol. Chem. Rapid Commun. 1991, 12, 717.

[6] Cviklinski, J.; Tajbakhsh, A. R.; Terentjev, E. M. Eur. Phys. J. E 2002, 9, 427.

[7] Yu, Y.; Nakano, M.; Ikeda, T. Nature 2003, 425, 145.

[8] Schätzle, J.; Kaufhold, W.; Finkelmann, H. Makromol. Chem. 1989, 190, 3269.

[9] Pakula, T.; Zentel, R. Makromol. Chem. 1991, 192, 2401.

[10] Gallani, J. L.; Hilliou, L.; Martinoty, P.; Doublet, F.; Mauzac, M. J. Phys. II Fr. 1996, 6, 443. 
[11] Zanna, J. J.; Stein, P.; Marty, J. D.; Mauzac, M.; Martinoty, P. Macromolecules 2002, 35, 5459.

[12] Zentel, R.; Ringsdorf, H. Makromol. Chem., Rapid Commun. 1984, 5, 393.

[13] Dubois, J. C.; Decobert, G.; Le Barny, P.; Esselin, S.; Friedrich, C.; Noël, C. Mol. Cryst. Liq. Cryst. 1986, 137, 349.

[14] Percec, V.; Tomazos, D.; Willingham, R. A. Polym. Bull. 1989, 22, 199.

[15] Percec, V.; Hahn, B. Macromolecules 1990, 23, 2092.

[16] Finkelmann, H.; Ringsdorf, H.; Wendorff, J. H. Makromol. Chem. 1978, 179, 273.

[17] Demus, D.; Zaschke, H. Flussige Kristalle in Tabellen II; VEB Deutscher Verlag für Grundstoffindustrie: Leipzig, 1984.

[18] Noirez, L. ; Keller, P. ; Cotton, J. P. Liq. Cryst. 1995, 18, 129.

[19] Zentel, R.; Reckert, G. Makromol. Chem. 1986, 187, 1915.

[20] Mitchell, G. R.; Davis, F. J.; Ashman, A. Polymer 1987, 28, 639.

[21] Warner, M.; Terentjev, E. M. Liquid Crystal Elastomers; Oxford University Press: Oxford, 2003.

[22] Zentel, R. Liq. Cryst. 1986, 1, 589.

[23] Barnes, N. R.; Davis, F. J.; Mitchell, G. R. Mol. Cryst. Liq. Cryst. 1989, 168, 13.

[24] Kishi, R.; Kitano, T.; Ichijo, H. Mol. Cryst. Liq. Cryst. 1996, 280, 109.

[25] Terentjev, E. M.; Warner, M.; Meyer, R. B.; Yamamoto, J. Phys. Rev. B 1999, $60,1872$.

[26] Urayama, K.; Kondo, H.; Arai, Y. U.; Takigawa, T. Phys. Rev. E 2005, 71, 051713.

[27] Yusuf, Y.; Huh, J. H.; Cladis, P. E.; Brand, H. B.; Finkelmann, H.; Kai, S. Phys. Rev. E 2005, 71, 061702.

[28] Shibaev, V. P.; Kostromin, S. G.; Plate, N. A. Eur. Polym. J. 1982, 18, 651.

[29] Galli, G. ; Chiellini, E. ; Laus, M. ; Caretti, D. ; Angeloni, A. S. Makromol. Chem., Rapid Commun. 1991, 12, 43.

[30] Kostromin, S. G.; Talroze, R. V.; Shibaev, V. P.; Plate, N. A. Makromol. Chem., Rapid Commun. 1982, 3, 803.

[31] Kasko, A. M.; Heintz, A. M.; Pugh, C. Macromolecules 1998, 31, 256.

[32] Kasko, A. M.; Grunwald, S. R.; Pugh, C. Macromolecules 2002, 35, 5466.

[33] Demus, D.; Richter, L. Textures of Liquid Crystals; VCH Publication: New York, 1978.

[34] Treloar, L. R. G. The Physics of Rubber Elasticity; Clarendon Press: Oxford, 1975.

[35] Erman, B.; Mark, J. E. Structure and Properties of Rubber-like Networks; Oxford University Press, New York, 1997.

[36] Brochard, F. J. Phys. (Paris) 1979, 40, 1049.

[37] Wang, X. J.; Warner, M. Macromol. Theory Simul. 1997, 6, 37.

[38] Matsuyama, A.; Kato, T. Phys. Rev. E 2001, 64, 010701.

[39] Benmouna, F.; Maschke, U.; Coqueret, X.; Benmouna, M. Polym. Int. 2001, 50, 469.

[40] Matsuyama, A.; Kato, T. J. Chem. Phys. 2002, 116, 8175.

[41] Urayama, K.; Okuno, Y.; Kawamura, T.; Kohjiya, S. Macromolecules 2002, 35, 4567.

[42] Urayama, K.; Okuno, Y.; Nakao, T.; Kohjiya, S. J. Chem. Phys. 2003, 118, 2903.

[43] Urayama, K.; Okuno, Y.; Kohjiya, S. Macromolecules 2003, 36, 6229.

[44] Yusuf, Y.; Ono, Y.; Sumisaki, Y.; Cladis, P. E.; Brand, H. R.; Finkelmann, H.; Kai, S. Phys. Rev. E 2004, 69, 021710.

[45] Vendamme, R.; Maschke, U. e-Polymers 2006, no. 069. 
[46] Vendamme, R. ; Bouchaour, T. ; Pakula, T. ; Coqueret, X. ; Benmouna, M. ; Maschke, U. Macromol. Mater. Eng. 2004, 289, 153.

[47] Dusek, K.; Patterson, D. J. Polym. Sci., Part A-2 1968, 6, 1209.

[48] Flory, P. J. Principles of polymer chemistry, Cornell University Press, Ithaca, 1965.

[49] Serbutoviez, C.; Kloosterboer, J. G.; Boots, H. M. J.; Touwslager, F. J. Macromolecules 1996, 29, 7690.

[50] Radiation curing in polymer science and technology, Fouassier, J. P.; Rabek, J. F. Eds., Elsevier Applied Science, London, 1993.

[51] Benmouna, F.; Peng, B.; Rühe, J.; Johannsmann, D. Liquid Crystals 1999, 26, 1655.

[52] Chiu, H. W. ; Zhou, Z. L. ; Kyu, T. ; Cada, L. ; Chien, L. C. Macromolecules 1996, 29, 1051.

[53] Kihara, H. ; Kishi, R. ; Kato, T. ; Ichijo, H. Polymer 2001, 42, 1177.

[54] Okuno, Y.; Urayama, K.; Kohjiya, S. J. Chem. Phys. 2003, 118, 9854.

[55] Kempe, M. D. ; Scruggs, N. R. ; Verduzco, R. ; Lal, J. ; Kornfield, J. A. Nat. Mater. 2004, 3, 177. 